\title{
Digital Component Separator for future W-CDMA-LINC Transmitters implemented on an FPGA
}

\author{
W. Gerhard and R. Knöchel \\ Microwave Laboratory, University of Kiel, Kaiserstrasse 2, 24143 Kiel, Germany
}

\begin{abstract}
This paper presents the implementation of a Digital-Component-Separator (DCS) for a LINC-transmitter (linear amplification using nonlinear components) on an FPGA (field programmable gate array). It investigates and estimates the bandwidth requirements for such a LINC system. The influence of bandwidth limitations on a digitally based LINC-transmitter for W-CDMA utilization is studied by simulations. Furthermore a LINC transmitter is proposed which employs a flexible image-reject- or a direct up-conversion-architecture for transmission of single or combined multi-carrier/channel W-CDMA signals using the phase-modulation approach. The sampling frequency can be chosen at a value up to 32 times $(122.88 \mathrm{MHz})$ the symbol rate of the W-CDMA chip rate of $3.84 \mathrm{Mbits} / \mathrm{s}$. Measurement results for a LINC transmitter are presented and discussed.
\end{abstract}

\section{Introduction}

In modern wireless communication systems as e.g. W-CDMA or W-LAN linearity and efficiency of the power amplifiers (PA) increasingly are becoming key parameters. The trade off between linearity and efficiency can not be solved with the mainly employed linearization methods like PA back-off, feedback, digital pre-distortion, feed-forward etc. Applying these methods admits to achieve the linearity requirements but yields a poor efficiency. With the application of modern digital signal processing, the LINC method becomes more attractive and interesting. It is promising the potential for solving the trade off in PA design. The LINC technique is based on a vector outphasing method introduced in Chireix (1935). An amplitude and phase modulated (AM/PM) signal is decomposed into two exclusively phase modulated (PM) signals with constant envelope. It is then amplified by two saturated power amplifiers operating with high efficiency. A final vector summation reconstructs the original AM/PM-signal. An important building block of such a complex LINC-transmitter is the component separator performing the signal decomposition. Suggested analog implementations suffer from insufficient realization

Correspondence to: W. Gerhard

(wg@tf.uni-kiel.de) of the required nonlinear mathematical operations (Shi and Sundstroem, 2000; Schemel, 1999). Implementations based on digital software do not offer the required bandwidth necessary for processing W-CDMA signals in real time (Hetzel et al., 1991). Thus a digital hardware design of the DCS (digital component separator) based on an FPGA may solve the problem and will be presented in this paper. The FPGA implementation provides the wide frequency bandwidth required for processing W-CDMA signals. The DCS is realized with an efficient hardware structure. It calculates the envelope and phase of the baseband signal and provides two phase modulated signals. The LINC technique is not only limited to one carrier applications as they occur e.g. in the uplink (mobile to base station). Our implementation is also capable of processing two ore more independent baseband channels. This is particularly interesting for the downlink (base station applications) where the transmission of several channels over a single PA may offer an economical advantage.

In the transmit stage four DDSs (direct digital synthesizers) capable of forming two different transmitter topologies together with an analog circuitry are implemented. If the interface to the analog transmitter circuit is DC-coupled the signals are directly up-converted with I/Q modulators. If AC-coupling is employed the DDSs carry out the necessary phase modulation on a first low IF. The resulting PM-signals are then up-converted using the same hardware. Therefore this image-reject architecture operates as a weaver-type (Razavi, 1998) up-converter. The achieved maximum bandwidth for this implementation is approximately $120 \mathrm{MHz}$ for direct up-conversion. After multistage amplification with saturated amplifiers the original signal is reconstructed using a rat-race coupler. The necessary correction of the amplitude- and phase imbalance caused by the analog hardware components is easily accomplished by the DDSs.

Furthermore this paper addresses the system bandwidth required for the amplification of the PM-signals. W-CDMA signals have large crest factors causing a virtually infinite PM-bandwidth. The thus required bandwidth truncation of the intended angle modulated signals leads to an undesired distorted AM/PM-modulation of them (Kammeyer, 1992). 


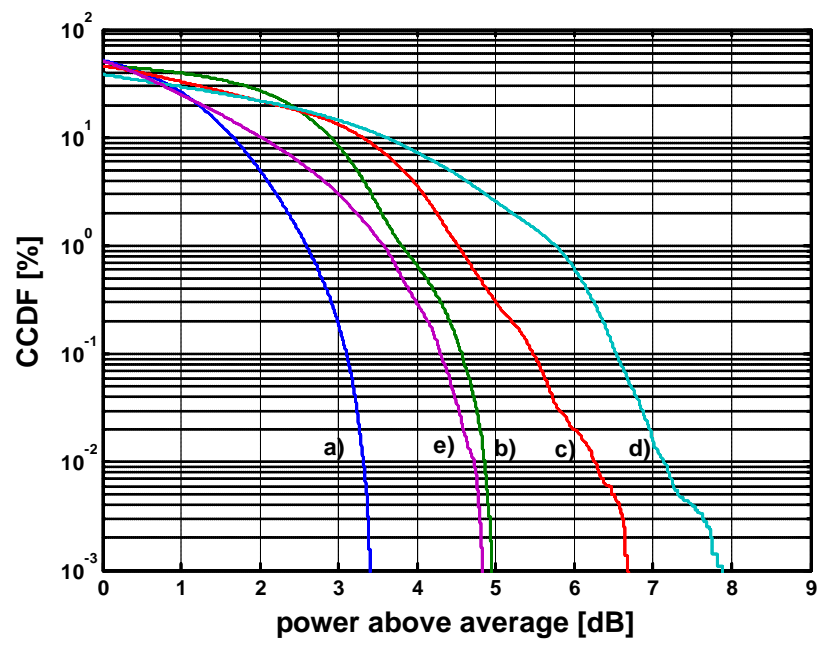

Fig. 1. Simulated CCDF-plots for a WCDMA uplink signal with different physical channel settings and for a $\mathrm{PN}$-code.

(a) $1 \mathrm{DPCCH}, 1 \mathrm{DPDCH}$ (data rate $=1 \times 960 \mathrm{kbps}$ )

(b) $1 \mathrm{DPCCH}, 2 \mathrm{DPDCH}$ (data rate $=2 \times 960 \mathrm{kbps}$ )

(c) $1 \mathrm{DPCCH}, 3 \mathrm{DPDCH}$ (data rate $=3 \times 960 \mathrm{kbps}$ )

(d) $1 \mathrm{DPCCH}, 6 \mathrm{DPDCH}($ data rate $=6 \times 960 \mathrm{kbps})$

(e) PN-Code

(a-d) with Spreading Factor=4, Gain=1, (DPCCH-Dedicated Physical Control Channel, DPDCH-Dedicated Physical Data Channel, (3GPP TS 25.213 V5.2.0 (2002-09), 3GPP TS 25.211 V5.2.0 (2002-09)).

The AM part hereof is opposing the original idea of LINC and can be removed by a limiter or by the PA itself which is operating in strong compression, thus avoiding distortions by intermodulation effects. Due to the remaining phase distortion the finally reconstructed signal will be distorted as well. The impaired vector cancellation results in increased ACPR-values (adjacent channel power ratio) and modulation distortions.

This paper is organized as follows: In Sect. 2 a short overview is given of the LINC-method and its utilization for a multi-carrier application. In Sect. 3 the influence of bandwidth limitations on phase modulated signals is investigated. Then simulation results for the application at a W-CDMA uplink (one channel) are presented and discussed. Section 4 focuses on the digital and analog hardware implementation. Measurement results are presented in Sect. 5, followed by final conclusions in Sect. 6 .

\section{LINC}

A band pass signal can be mathematically represented as

$S(t)=\operatorname{Re}\left[A_{1}(t) e^{j \theta(t)}\right]$

with $0 \leq A_{1}(t) \leq A_{\max } ; \theta(t)=\omega t+\varphi(t)$.

That amplitude- and phase-modulated signal can be separated into two constant envelope phase modulated portions, which may be described by the phase modulation method (Zhang et al., 2003). The phase modulation method directly modulates the phase of two LINC carriers and yields two phase modulated signals as

$$
\begin{aligned}
& S(t)=S_{L I N C_{-1}}(t)+S_{L I N C_{2} 2}(t) \\
& S_{L I N C_{-} 1}(t)=A_{1 C} \cos \left(\omega t+\varphi(t)+\arccos \left(A_{1}(t) / A_{\max }\right)\right) \\
& S_{L I N C_{-} 2}(t)=A_{1 C} \cos \left(\omega t+\varphi(t)-\arccos \left(A_{1}(t) / A_{\max }\right)\right)
\end{aligned}
$$

with $A_{1 C}$ - Amplitude of the single LINC-carrier, $A_{\max }-$ peak amplitude of the envelope.

From Eq. (1) it is easily deduced that the expression is generally true and can be applied also to the sum of various independent bandwidth-limited AM/PM-Carriers. In general the I- and Q- components of one baseband channel are used to modulate one carrier signal. Adding more baseband channels requires additional carriers. In order to apply the LINC method to multi-carrier/channel signals the case of two baseband channels will be considered. Two AM/PM-carriers are combined in the digital complex baseband domain and yield

$S_{2 C}(t)=B B_{1}(t)+B B_{2}(t)$

$S_{2 C}(t)=a_{1}(t) e^{j \varphi_{1}(t)} e^{j \omega_{1} t}+a_{2}(t) e^{j \varphi_{2}(t)} e^{j \omega_{2} t}$

The amplitude- and phase-information can be expressed through the respective I/Q-components

$S_{2 C}(t)=\left[I_{1}(t)+j Q_{1}(t)\right] e^{j \omega_{1} t}+\left[I_{2}(t)+j Q_{2}(t)\right] e^{j \omega_{2} t}(4)$

This leads to a necessarily complex modulation in the baseband. For one modulated channel one obtains

$$
\begin{aligned}
& B B_{1}(t)=\operatorname{Re}(t)_{B B 1}+j \operatorname{Im}(t)_{B B 1}, \text { with } \\
& \operatorname{Re}(t)_{B B 1}=\left[I_{1}(t) \cos \left(\omega_{1} t\right) \mp Q_{1}(t) \sin \left(\omega_{1} t\right)\right] \\
& \operatorname{Im}(t)_{B B 1}= \pm j\left[I_{1}(t) \sin \left(\omega_{1} t\right) \pm Q_{1}(t) \cos \left(\omega_{1} t\right)\right]
\end{aligned}
$$

Equation (5) shows the property of a complex modulation, that the imaginary part is the Hilbert-transform of the real part. Such an "analytical" signal contains frequency components only on one side of the frequency axis. The \pm signs in Eq. (5) define which image on the positive or negative part of the frequency axis will be extinguished. Adding two baseband channels having complex modulation leads to

$S_{2 C}(t)=\left[\operatorname{Re}(t)_{B B 1}+\operatorname{Re}(t)_{B B 2}\right]+j\left[\operatorname{Im}(t)_{B B 1}+\operatorname{Im}(t)_{B B 2}\right](6)$

This procedure can easily be extended to $\mathrm{N}$ independent channels having different modulations (I/Q-contents). For the resulting signal Eq. (6) the classical LINC-approach can be applied. It is thus not limited to only one AM/PM carrier.

\section{$3 \quad$ PM - Bandwidth Limitation} 3.1 Signal Statistics of W-CDMA uplink and
PM-Bandwidth

The W-CDMA system specifications are described in 3GPP TS 25.213 V5.2.0 (2002-09), 3GPP TS 25.211 V5.2.0 


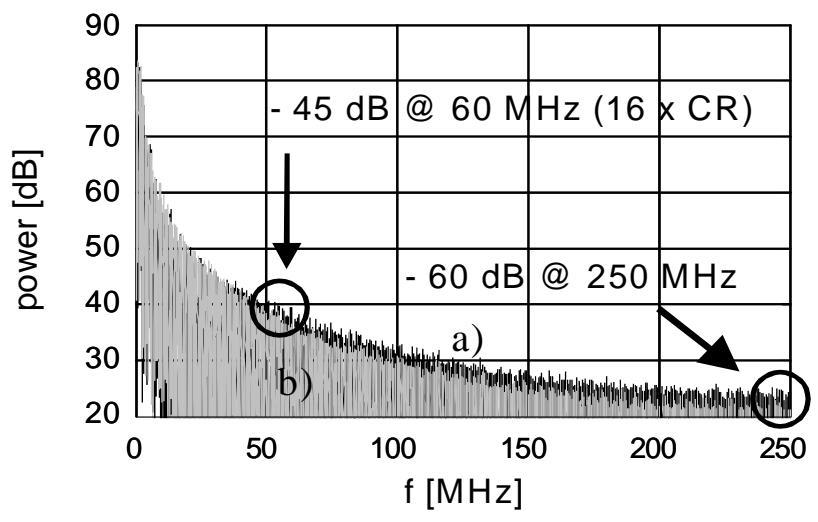

Fig. 2. Simulated spectra of the phase modulated LINC signal derived for two different W-CDMA uplink signals with different crest factors. (a) crest: $3.5 \mathrm{~dB}(960 \mathrm{kbps})$, (b) crest: $8 \mathrm{~dB}$ $(6 \times 960 \mathrm{kbps})$.

(2002-09), and 3GPP TS 25.101 V5.2.0 (2002-03). The utilized CDMA coding can handle different data rates and maps them always to a constant chip rate of $3.84 \mathrm{Mchips} / \mathrm{s}$ using DS-SS (direct sequence-spread spectrum). From the PA point of view these different data rates results in different AM-statistics. The signal statistics of a W-CDMA uplink signal can be described by the Complementary Cumulative Power Distribution Function (CCDF). Figure 1 plots the CCDF for a W-CDMA uplink signal with different physical channel settings. As can be seen the crest factors are in the range between 3.4 to $8 \mathrm{~dB}$ (given by the intersection of the curves with the horizontal axis). For measurements a pseudo-noise-source was used, which has a crest factor of nearly $5 \mathrm{~dB}$ (curve 1e). Figure 2 shows the simulated frequency spectra of the phase modulated constant envelope LINC signal after signal decomposition. It can be seen that the spectra for two different uplink cases with different AM-statistics (crest: 3.5/8 dB) are nearly identical. Most of the power is concentrated around the carrier $(\mathrm{f}=0 \mathrm{MHz})$ and then rapidly drops down at higher frequency offset. At a frequency offset of $60 \mathrm{MHz}$ (about $16 \times$ the chip rate, $\mathrm{CR}$ ) a spectral attenuation of about $-45 \mathrm{~dB}$ occurs and at $250 \mathrm{MHz}$ the attenuation is approximately $-60 \mathrm{~dB}$.

It can be supposed that the required PM bandwidth does not depend on the pay load (data rate) and resulting crest factor, respectively.

\subsection{Bandwidth limitations and system model}

The resulting spectral distribution is mainly defined by the digital sampling frequency and through the construction of the required analog reconstruction filters, which depends on the sampling frequency. The actually infinite frequency bandwidth has to be truncated, which results in a distorted AM/PM time function and an overlapping of remaining aliasing frequency components. The proper choice of the sampling frequency defines the requirements on the digital components and the power consumption. Therefore different
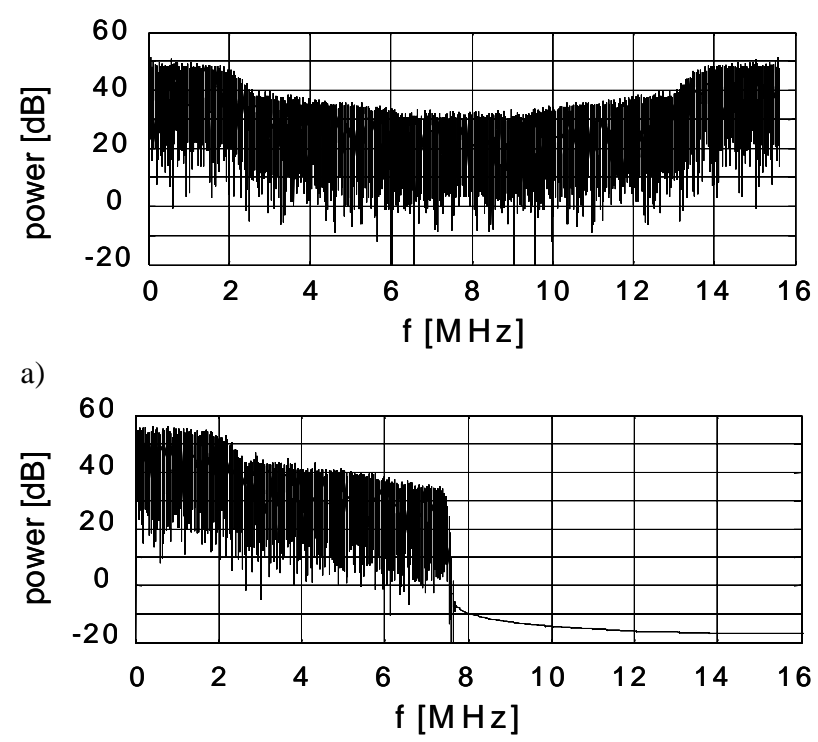

b)

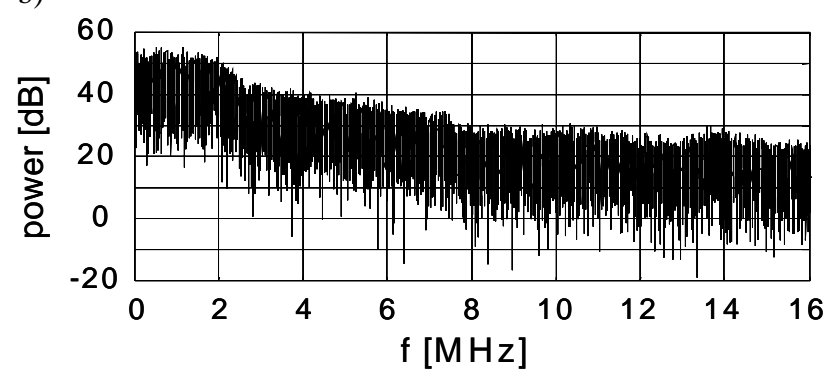

c)

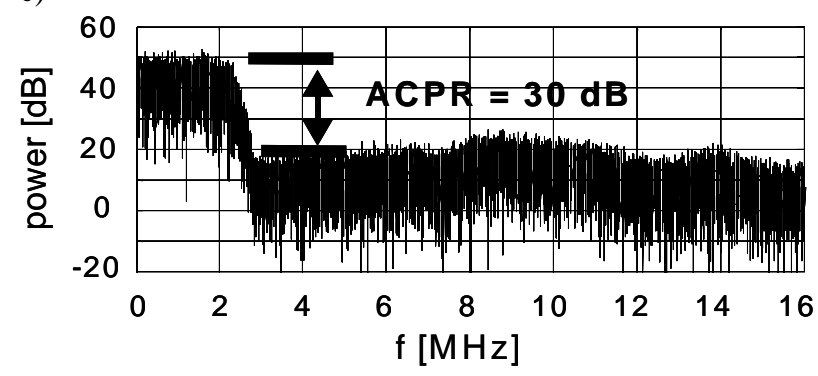

d)

Fig. 3. Simulated spectra of the phase modulated LINC signal, crest $=8 \mathrm{~dB}$, f_sampling $=4 \times \mathrm{f}$ _chip $(15.36 \mathrm{MHz})$, (a) $\mathrm{PM}-\mathrm{LINC}$ signal with periodical spectrum, (b) after DAC and reconstruction filtering, (c) after limiter, (d) after signal reconstruction.

system simulations were performed in order to estimate the practical requirements on frequency bandwidth and their influence on the amount of introduced signal distortions in terms of ACPR and modulation distortions. After taking AM/PM-signals with different signal statistics and various crest factors as a basis the LINC algorithm is applied. The data of the thus generated corresponding PM-signals then are digital- to analog- converted using the $\sin x / x$-shaping characteristics of the zero-order-hold DAC's and are then band limited through low pass filtering with nearly ideal rectangular reconstruction filters. Any resulting AM is 


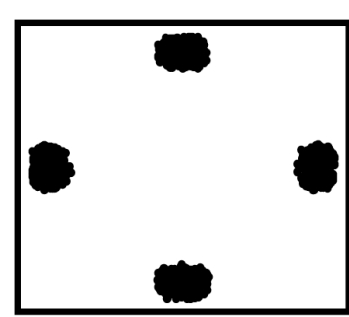

a)

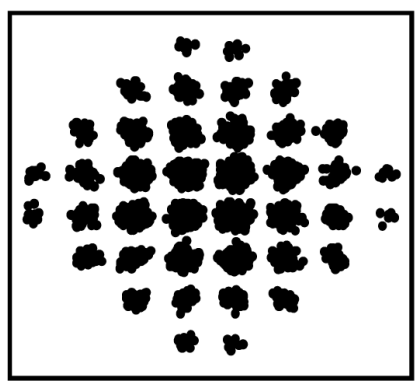

b)
Fig. 4. Simulated I/Q modulation distortions over 3000 symbols for f_sampling $=4 \times \mathrm{f}_{\text {_chip }}(15.36 \mathrm{MHz}), \quad$ (a) crest $=3.5 \mathrm{~dB}, \quad$ (b) crest $=8 \mathrm{~dB}$.

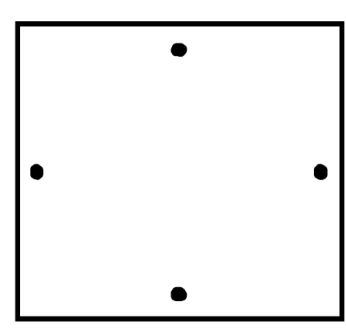

a)

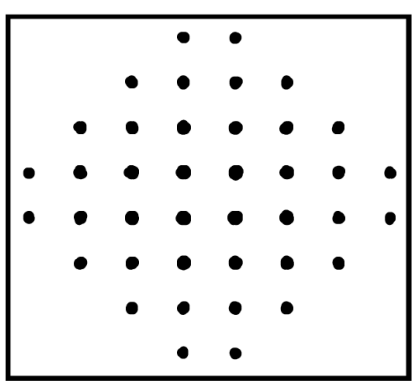

b)
Fig. 5. Simulated I/Q modulation distortions over 3000 symbols for f_sampling $=32 \times \mathrm{f}_{\text {_chip }}(122.88 \mathrm{MHz})$, (a) crest $=3.5 \mathrm{~dB}$, (b) crest $=8 \mathrm{~dB}$.

removed by an amplitude limiter. The addition of the two (now distorted) PM signals reconstructs the original (now distorted) AM/PM signal. The LINC-algorithm and the DAC's operate at the system sampling frequency.

\subsection{Simulation results}

For the investigation of the influence of spectral aliasing and bandwidth truncation simulation results are presented in Fig. 3 as examples. Because the sampling frequency ( $4 \times$ chip rate) is too low, aliasing of the periodical spectra occurs (Fig. 3a). After removing of the AM with a limiter (Figs. 3b, c) PM-errors are induced, which lead to a poor vector cancellation and to a poor ACPR value of the final reconstructed signal (Fig. 3d). For evaluating the in band distortions and their impact on the modulation accuracy the recombined and demodulated signal is taken into consideration. W-CDMA uses a special HPSK-modulation method, which has the property of always reducing the crest factors due to the modulation itself (Designing and testing 3GPP W-CDMA user equipment). However only for the special case mentioned in Fig. 1a (data rate $=1 \times 960 \mathrm{kbps}$ ) a QPSK-plot rotated by $45^{\circ}$ results. If the data rate is further increased (Figs. 1b-d) higher order I/Q constellation plots are obtained, reducing the bit error rate for same distortions.

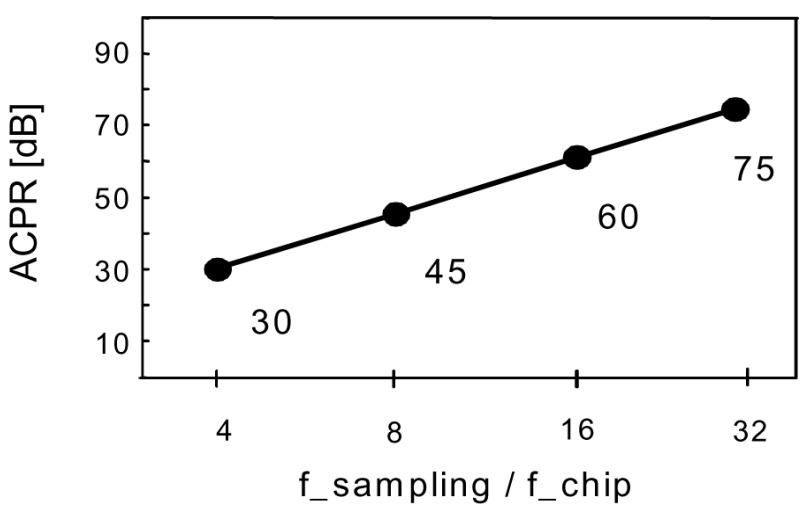

Fig. 6. Simulated ACPR as function of the sampling frequency and the system chip rate.

Figure 4 demonstrates the I/Q distortion due to bandwidth restriction and AM-limitation for two signals with different crest factors. The two plots (Figs. 4a, b) were obtained for a sampling frequency of four times the chip rate. It can be deduced from these graphs that amplitude- and phase errors occur after signal reconstruction. This indicates that the selected sampling frequency is too low. That conclusion correlates with the obtained poor ACPR-value of about $30 \mathrm{~dB}$ (Fig. 3d). If the sampling frequency and thus the bandwidth is increased the ACPR values and I/Q modulation distortions are reduced. In comparison with the cases of Fig. 4 the graphs of Fig. 5 plot the I/Q charts for a sampling frequency selected much higher, namely of $32 x$ the chip rate $(122.88 \mathrm{MHz})$. Now the I/Q distortions are very small, indicating a sufficient bandwidth for the transmission of the PM-signals. The corresponding ACPR value for this case is approximately $75 \mathrm{~dB}$. The simulated ACPR-values are plotted in Fig. 6 as function of the ratio of the sampling frequency and the system chip rate of 3.84 Mchips/s.

The W-CDMA uplink system specification is defined through a minimum of the ACLR (Adjacent Channel Leakage Ratio) which is specified to be $-33 \mathrm{~dB}$ at $5 \mathrm{MHz}$ offset and $-43 \mathrm{~dB}$ at $10 \mathrm{MHz}$ offset (3GPP TS 25.101 V5.2.0 (2002-03)). Considering Fig. 6 and taking an additional system margin into account a sampling frequency between $8 \times(30.72 \mathrm{MHz})$ and $16 \times(61.44 \mathrm{MHz})$ the chip frequency has to be selected. This frequency range is quite interesting for digital signal processing because circuits with low power consumption for mobile applications may be realized in that range, making a deployment of the LINC technique possible.

\section{Digital and analog Hardware Implementation}

\subsection{FPGA implementation}

The DCS was implemented on a XILINX Virtex2-1500 FPGA (Xilinx datasheet, 2001). The used FPGA platform is a Xilinx-Virtex 2 prototype board provided with a special 


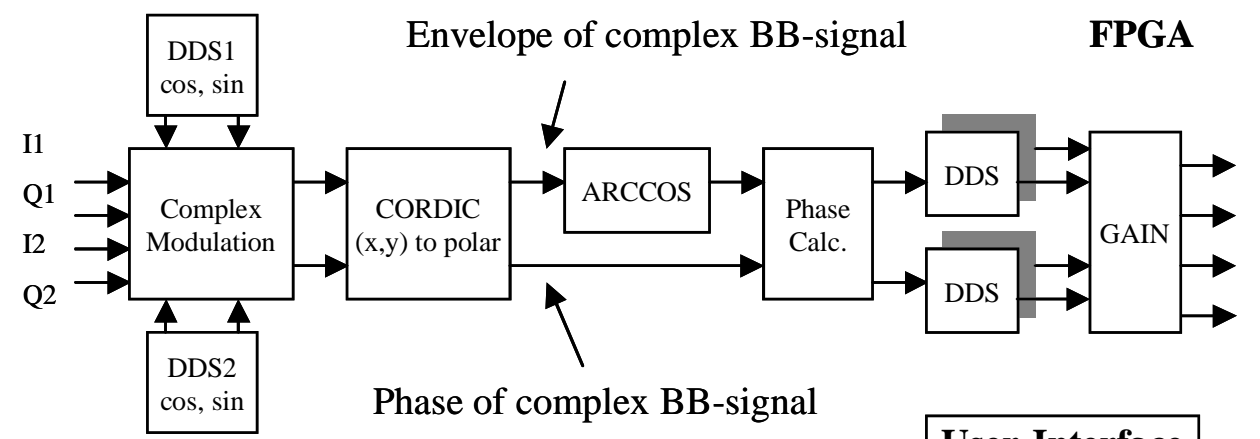

User-Interface

Fig. 7. FPGA implementation of the LINC algorithm.

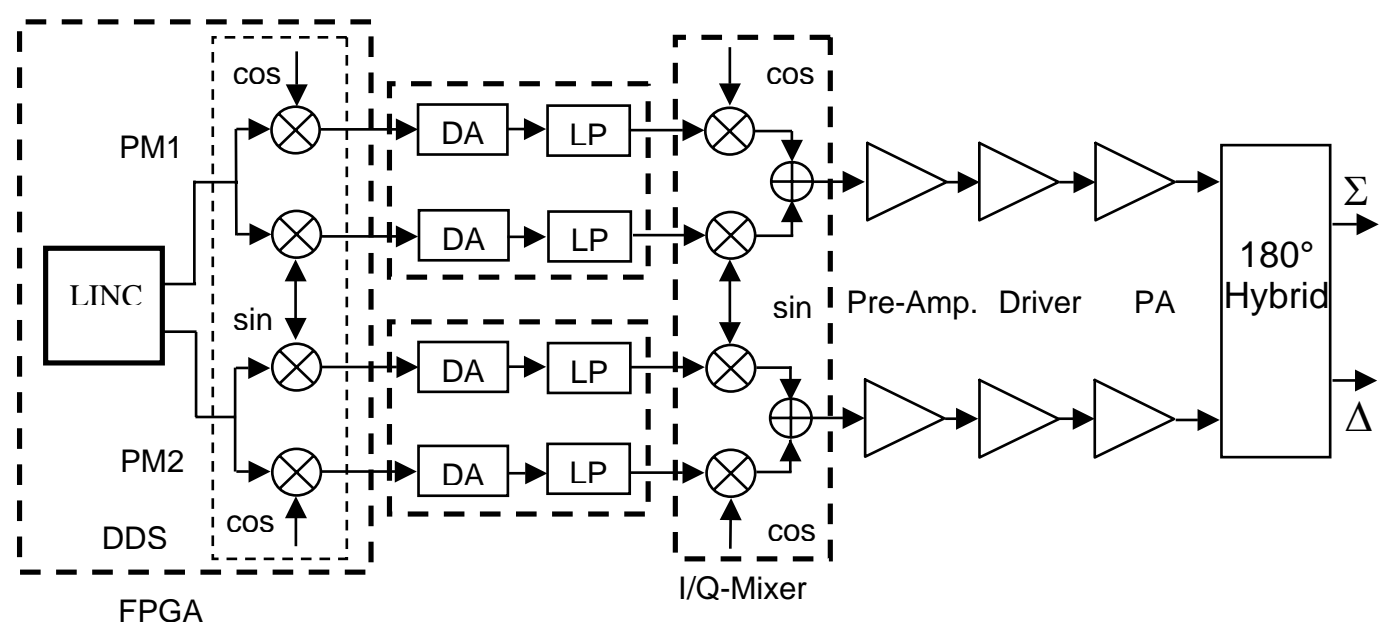

Fig. 8. Schematic of the entire LINC transmitter.

chip socket. This socket can carry Virtex2-FPGAs with different logic sizes (XC2V1500... XC2V3000) and is quite practical for adaptation of different system requirements. The addressable resources of a Xilinx-FPGA are RAM-based look-up tables (LUT) which can be configured as a shiftregister, as well as RAM or ROM, Multiplexers, FlipFlops, BLOCK RAM and special dedicated hardware multipliers for high speed applications. The fixed point hardware was designed and simulated with a hardware design language (VHDL) using the design package FPGA Advantage from Mentor Graphics. Some baseband components like filters were designed, simulated and optimized using ADS from Agilent Technologies. The system clock frequency is $122.88 \mathrm{MHz}(32 \times 3.84 \mathrm{MHz})$ and is internally divided down to different clock domains.

\subsection{Implementation of the LINC algorithm}

Figure 7 displays a schematic overview of the implementation of the LINC algorithm. The interpolated I- and Q-signals of each channel are complex modulated. The cosine and sine operations are performed by using a DDS structure (Razavi,
1998). The frequency allocation of the baseband channels is set by loading a tuning word to the DDSs. The resulting real and imaginary parts of the complex modulated signals are then fed to a special CORDIC (COordinate Rotational DIgital Computer)-algorithm (Volder, 1959) as (x,y)-vector information. This algorithm calculates the amplitude- and phase of a (x,y)-vector and can be regarded as a rectangular to polar vector translation. The CORDIC thus derives the final envelope and phase of the two combined baseband channels. After these steps the LINC method can be applied. The necessary arccos-function is stored as a LUT in RAM. Finally the calculated phase signals for the LINC are used for phase modulation of four DDSs at a carrier frequency between 0 and $1 / 4$ of the sampling frequency.

The weaver image-reject architecture is provided by tuning the offset-phases of the DDSs. They are also adjusted for compensation of the branch phase-imbalances. For a direct up-conversion system (DC-coupled) the IF-frequency of the DDSs is set to zero. Gain control is provided for the compensation of branch amplitude-imbalances. 


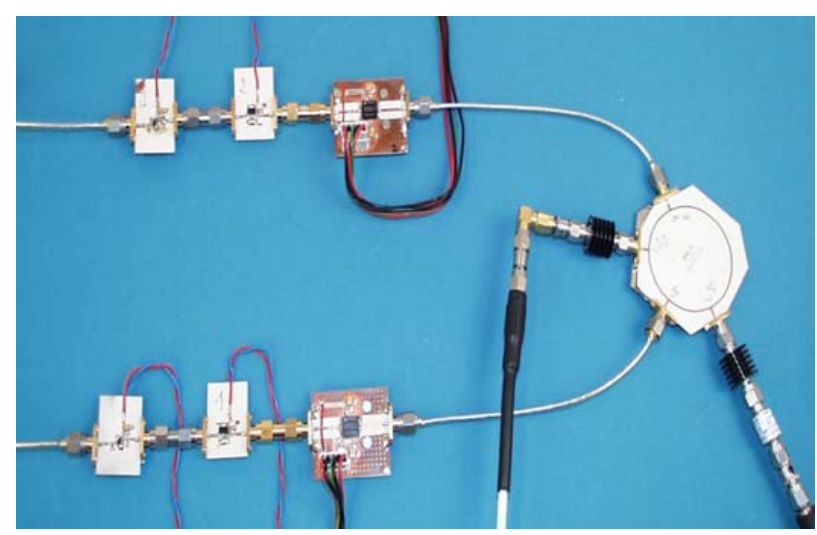

Fig. 9. Multistage amplifiers and signal combining set up.

\subsection{LINC transmitter}

The entire transmitter set up is shown in Fig. 8. After digital to analog conversion and low pass filtering the signals are supplied to two double-balanced active I/Q-mixers (Mod. Bandwidth: $70 \mathrm{MHz}$, Analog-Devices) and up-converted to a center frequency of $2 \mathrm{GHz}$. The four branches were adjusted by cut and try at a single frequency because of the lack of a closed-loop calibration algorithm. Two linear power amplifiers (CX65101) from Skyworks driven into compression were applied. The gain was $21 \mathrm{~dB}$ and the measured $1 \mathrm{~dB}$ compression point was approximately $30 \mathrm{dBm}$. A MMIC amplifier (GAL51, $\mathrm{G}=16.5 \mathrm{~dB}$, Minicircuits) and a preamplifier (SGA-0363, $\mathrm{G}=17.2 \mathrm{~dB}$, Sirenza) were provided for driving the power amplifiers. Each amplification stage was impedance matched to $50 \mathrm{Ohm}$ and constructed on a single printed circuit board with SMA-connectors. An advantage of the LINC is that the drivers can also be driven into compression. Thus the efficiency is improved. The generated harmonics have to be suppressed before entering the next stage. That task is automatically fulfilled by the low pass matching circuits at the amplifier inputs and outputs. Both LINC-signals are combined with a $180^{\circ}$ microstrip rat race hybrid coupler. The SMA-connectors and semi-rigid cables connecting the power amplifiers lead again to an increase of the branch imbalances. The multistage amplifier set up with its hybrid signal combining is depicted in Fig. 9.

\section{Measurement results and discussion}

In this section experimental results and measurements will be presented and discussed for the set up applying the LINC algorithm. Measurements were made at a center frequency of $2 \mathrm{GHz}$ and not at the originally dedicated uplink/downlink frequencies (3GPP TS 25.101 V5.2.0 (2002-03)). For the ACPR measurements a FSQ-Spectrum/Vector-SignalAnalyzer from Rohde \& Schwarz has been used. For the

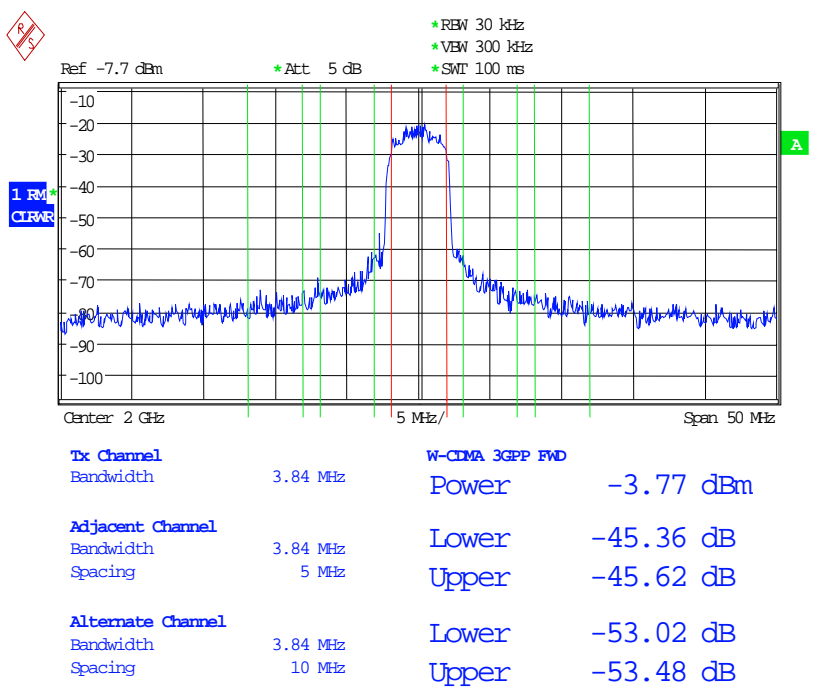

Fig. 10. Frequency spectrum of a W-CDMA-uplink signal after hybrid combining.

following measurements the constant phase offset between the two LINC channels had to be removed. That was achieved by loading phase offset values to the DDSs. Efforts aiming at the adjustment of the amplitude imbalances for improving the results were not successful, indicating that the PA's were driven in strong compression. Hence the different branches were driven with the same amplitudes and can be considered as being not adjusted.

\subsection{One W-CDMA uplink channel, direct up-conversion}

Firstly the performance of the case where one channel is transmitted by using the direct up-conversion transmitter architecture was investigated. The provided bandwidth was about $120 \mathrm{MHz}$. Figure 10 shows a frequency plot for one W-CDMA channel with a corresponding ACPR of about $-45 \mathrm{~dB}$ for adjacent channels and $-53 \mathrm{~dB}$ for the alternate channels. This result implies that the system requirements (Sect. 3.3) are fulfilled and the bandwidth is broad enough to transmit the PM-LINC signals without distortions. The non perfect signal cancellation can be explained by the used calibration which is correct for only a single adjusted frequency. Better results may be obtained by applying a broadband calibration technique or channel pre-distortion.

5.2 One W-CDMA uplink channel, weaver image-reject up-conversion, $\mathrm{IF}=30 \mathrm{MHz}$

For the case of AC-interfacing a low IF weaver image-reject transmitter architecture can be employed without any hardware changes. A disadvantage of such structure is the restriction to half the frequency bandwidth. An investigation of the impact of bandwidth reduction on the PM-LINC signals from about $120 \mathrm{MHz}$ to $60 \mathrm{MHz}$ in this case is however interesting. The transmitter DDSs were adjusted to a first IF at the center frequency of $30 \mathrm{MHz}$ and the $\mathrm{LO}$ 


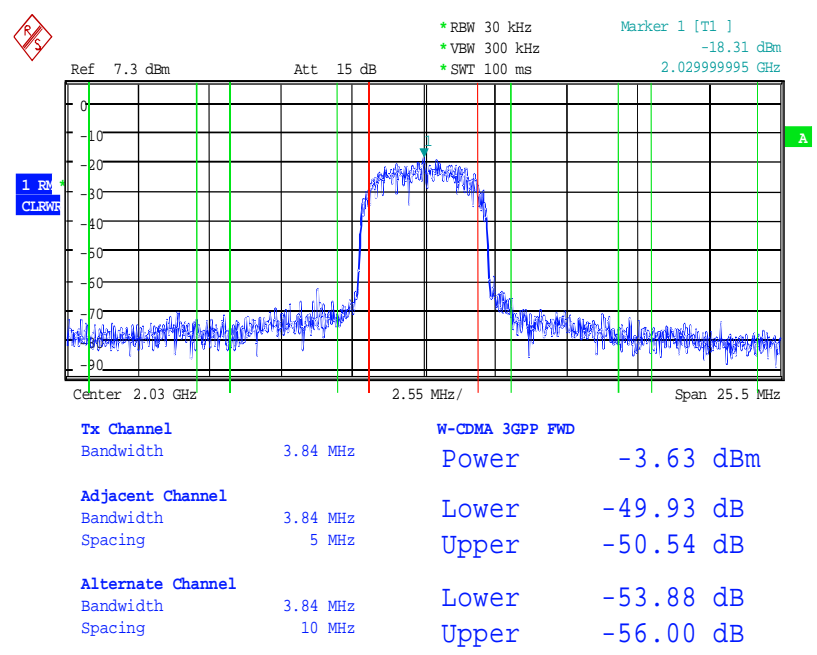

Fig. 11. Frequency spectrum of a WCDMA-uplink signal after hybrid combining, $\mathrm{IF}=30 \mathrm{MHz}$.

was fixed to $2 \mathrm{GHz}$. Therefore the channel was up-converted to a rf center frequency of $2.03 \mathrm{GHz}$. An ACPR plot is shown in Fig. 11. Compared to direct up-conversion even better results across the frequency span could be obtained. This is explained by a better hardware amplitude- and phase balance at this center frequency. An ACPR of about $-50 \mathrm{~dB}$ for adjacent channels and better than $-53 \mathrm{~dB}$ for the alternate channels was observed. The image suppression is about $50 \mathrm{~dB}$.

As a conclusion and taking the single channel uplink application into account, it can be derived that a sampling frequency of $16 \times$ the chip rate is enough to fulfill the requirements for W-CDMA. This was already estimated and predicted by simulations. An ACPR value of about $-60 \mathrm{~dB}$ was simulated as the best case situation (see Fig. 6). These results indicate that half the frequency bandwidth is sufficient. As a consequence the clock frequency of the digital part (FPGA, DAC) can be reduced resulting in a much lower power consumption of this part of the circuit.

\subsection{Two W-CDMA channel, direct up-conversion}

Finally the capability for the transmission of two independent channels over a single LINC transmitter by applying two carriers having a frequency spacing of $10 \mathrm{MHz}$ and a power difference of $6 \mathrm{~dB}$ was investigated. Figure 12 shows the plot of the ACPR measurements. The ACPR measurement for the carrier at the higher frequency delivers a value of approximately $-44 \mathrm{~dB}$ (upper/lower) which is comparable to the case investigated first (single carrier). The upper adjacent channel ACPR is about $-42 \mathrm{~dB}$. Both channels could be demodulated without any problems. The bandwidth requirement for the case of transmission of two simultaneous channels is much higher than for a single channel application and has to be investigated further in future work. However the presented results are a proof

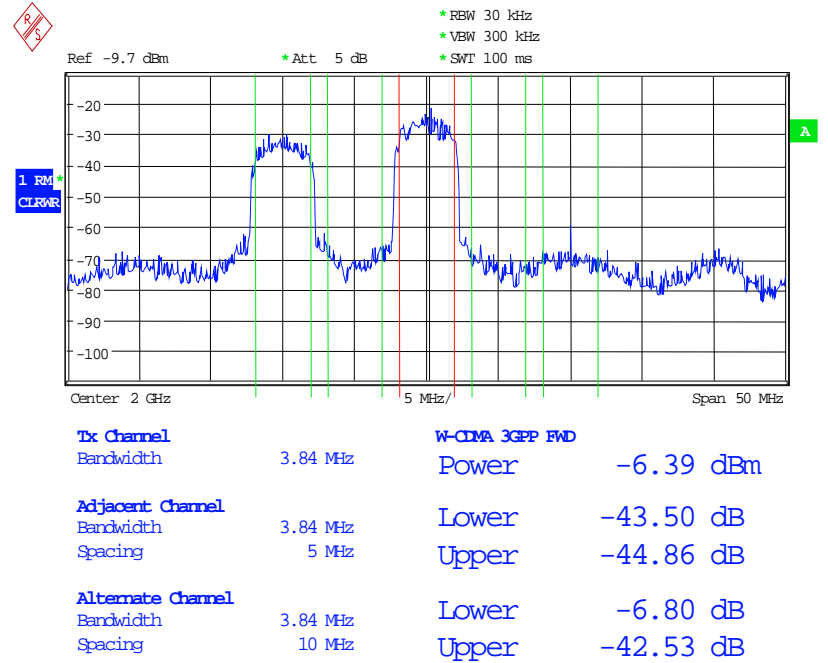

Fig. 12. Frequency spectrum of two WCDMA signals with $10 \mathrm{MHz}$ frequency spacing after hybrid combining.

for the LINC techniques capability of transmitting several independent channels simultaneously.

\section{Conclusion}

The demands on a LINC transmitter for a W-CDMA uplink application were investigated and specified in terms of required digital sampling frequency, bandwidth and attainable ACPR values. It was shown that for a single channel application the required PM-bandwidth virtually does not depend on the signal's amplitude statistics (crest). The impact of bandwidth limitations on the PM generated by a DCS was investigated in dependence of the sampling frequency and the shape of the analog reconstruction filter, if a generated $\mathrm{AM}$ is removed by a limiter. A digital component separator for the LINC transmitter was implemented through a fast digital hardware (FPGA) employing a maximum bandwidth of about $120 \mathrm{MHz}$. Furthermore a novel W-CDMA multi-channel transmitter using LINC was proposed. A flexible image-reject architecture for the upconversion of a single channel and/or for multiple channel utilization and for AC or DC coupled systems was described. Good performance regarding linearity, ACPR and image-rejection was obtained. Single/multi-channel transmission using LINC may also receive interest for an application in base-stations.

\section{References}

Chireix, H. : High power outphasing modulation, Pro. IRE, vol. 23, no. 11, 1370-1392, Nov. 1935.

Designing and testing 3GPP W-CDMA user equipment, Application Note 1356, available: http://www.agilent.com/find/3G, Agilent Technologies Inc., USA. 
Hetzel, S. A., Batema, A., and McGeehan, J. P.: LINC transmitter, Electronics Letters, vol. 27, no. 10, 884-845, May 1991.

Kammeyer, K. D.: Nachrichtenübertragung (in German), Stuttgart, Teubner, 1992.

Razavi, B.: RF Microelectronics, Prentice-Hall. INC., 1998.

Schemel, R. E.: Generating arcsine(x) and alternate method for LINC, Electronics Letters, vol. 35, no. 10, 782-783, May 1999.

Shi, B. and Sundstroem, L.: A 200-MHZ IF BiCMOS signal component separator for linear LINC transmitters, IEEE Journal of Solid-State Circuits, vol. 35, no. 7, 987-993, July 2000.

3GPP TS 25.213 V5.2.0 (2002-09): Spreading and modulation (FDD), Available: http://www.3gpp.org.

3GPP TS 25.211 V5.2.0 (2002-09): Physical channels and mapping of transport channels onto physical channels (FDD), Available: http://www.3gpp.org.

3GPP TS 25.101 V5.2.0 (2002-03): UE radio transmission and reception (FDD), Available: http://www.3gpp.org.

Xilinx datasheet, Virtex-II $1.5 \mathrm{~V}$ field-programmable gate arrays, XilinxInc., San Jose, CA, 2001, Available: http://www.xilinx. com.

Volder, J.: The CORDIC trigonometric computing technique, IRE Trans. Electronic Computing, vol. EC-8, 330-334, September 1959.

Zhang, X., Larson, L. E., and Asbeck, P. M.: Design of linear rf outphasing power amplifiers, Norwood: Artech House INC., 2003. 